\title{
QoS routing mechanism for MANET to support real-time service
}

\author{
Guangshuai Ding ${ }^{1,}$ a, Weimin Lei $^{1, b}$, Zhengxu $\mathrm{Hu}^{2}$, Zheyang Zhang ${ }^{1}$ \\ ${ }^{1}$ School of Computer Science and Engineering, Northeastern University, Shenyang, 110819, \\ China \\ ${ }^{2}$ School of Software, Northeastern University, Shenyang, 110819, China \\ aemail:664734781@qq.com, bemail: leiweimin@ise.neu.edu.cn
}

Keywords: multi-media service; MANET; QoS routing; AODV

\begin{abstract}
The development and widespread use of real-time constrained multi-media application in MANET has resulted in the need to optimize or update current routing mechanisms. This paper proposed a QoS routing mechanism for multi-media transmission under real-time constraint in MANET, named ad-hoc on-demand distance vector routing based on location information and linkstability evaluation (LL-AODV). The mechanism abstracts the routing problems into the shortest path problem of an undirected weighted graph in which the weights of the paths are determined by the duration time of links, the residual battery and energy consumption of nodes, and utilizes the shortest path algorithm to determine a feasible transport path. We use a prediction model of the duration time of links to predict the probable time the link may maintain, and that improves the deficiency of the AODV routing mechanism, which only uses hop counts as the transport path metric. Furthermore, it adopts periodical maintenance and rescue maintenance to ensure an alternative path can be found in time to transmit data, and thus improves the reliability of multimedia data transmission under real-time constraint. Simulation and the experimental results showed that the mechanism could provide a better QoS route for the multi-media transmission under realtime constraint in MANET.
\end{abstract}

\section{I . Introduction}

Mobile ad hoc network (MANET) [1][2] is a subset of wireless and mobile network. It is a multihop temporary autonomous system without fixed infrastructures, and it employs distributed protocols to maintain the network connection. All nodes in MANET are equal and mobile, and there is no central control node. Each node can communicate with an access point with its wireless transmitters and receivers. MANET has the properties of high networking speed and high survivability [3], which make it widely used in military, civil and other fields.

In recent years, with the development of multimedia and MANET technology, there is a great require of the real-time communication of multimedia data in MANET, such as real-time audio and video communication, etc. However, because of the characteristics in the MANETs, such as limited channel bandwidth, highly dynamic network topology, imprecise link state information, limited power at the nodes, etc. [4], the paths connection between source nodes and destination nodes may be very unstable and will go down at any time. It makes communication over MANET difficult and is hardly to assure the reliability of data transmission, much less could the protocol provide a better quality of service (QoS). To overcome these issues, appropriate cross-layer cooperation is required. QoS schemes provide QoS information by factoring the impacts of node mobility and lower-layer link parameters into QoS performance. But in the real-time multimedia transmission scenarios where the high rate and severe delay constraints are required, the above issues become more severe. Therefore, a new QoS routing mechanism performed well at the end-to-end real-time communication issues will have a practical significance and value.

Based on the summary of the existed researches, this paper puts forward a new QoS routing mechanism that works in the end-to-end real-time scenario - ad-hoc on-demand distance vector routing based on location information and link-stability evaluation (LL-AODV). LL-AODV introduces a prediction model of the duration time of links to predict the probable time the link may 
maintain, and abstracts the QoS routing problems into the shortest path problem of an undirected weighted graph. The implementation of the mechanism has reduced the time of transport path construction and improved the quality of transport paths. And the employment of routing maintenance mechanism which includes periodicity maintenance and rescue maintenance will further ensure the paths effectiveness and the data transport reliability.

The reminder of this paper is organized as follows. The related work will be introduced in Section II and Section III details the routing mechanism we proposed. Section IV presents simulations comparing our protocol with the conventional AODV. And Section V draws the conclusions and the future works.

\section{Related works}

\section{A. Overview of MANET routing}

In MANET, routing is always a challenging problem and there are varieties of MANET routing algorithms have been proposed. Numbers of MANET routing surveys have been published. Based on the methods of obtain routing information, MANET routing can be divided into table-driven routing and demand-driven routing. Hong et al. [5] is the oldest but still provides a good overview of the basic concepts, it divides routing into three categories, i.e., flat routing, hierarchical routing, geographic position assisted routing. And more detailed surveys have been published by Boukerche et al. [6] as well as Alotaibi and Mukerjee [7]. Mojamed et al. [8] summarized predecessor's research results and introduced eight categories: proactive routing, reactive routing, hybrid routing, hierarchical routing, multipath routing, multicast routing, geographical routing, geocast routing. However, among these routing categories, only a few researches focused on the real-time communication, especially the end-to-end real-time conversation.

The research of A. H. Marwa, et al. [9] showed that OLSR was not applicable for the real-time video service transport due to the limitation of the table-driven routing, but AODV could work well in the real-time transport scenario with a large range. And Huang et al. [10] research showed that by utilizing a revised AODV routing algorithm, the service of real-time transmission is considered viable in MANET. Therefore, by revising the conventional AODV, an end-to-end real-time transmission service for multi-media could be achieved on the basis of the corresponding increase in routing generation mechanism in MANET.

\section{B. AODV}

AODV [11] is a widely used routing algorithm in MANET. When data need to be transferred, the source node will check its router table first. If it matches a route item, the source node will send the data to its next hop. If the route item cannot be found, source node will broadcast RREQ control packet to its neighbor node and the nodes will establish or update a reserve routing when a nonrepetitive RREQ packet is received. The speed and the coordinates of the nodes can be obtained by utilizing Global Position System (GPS). All nodes pick up the RREQ packet and broadcast it until the destination node or the intermediate node which has a valid routing access to the destination node receives the packet. Then the destination node or the intermediate node will reply a RREP packet to source along the reverse path. The AODV adopts the first arrival RREQ to build a route when other routes have same hop count.

There's a ton of research on AODV routing mechanism. Experimental result of Setiadi et al. [12] showed that average delay of AODV routing mechanism was $0.253 \mathrm{~ms}$ and average throughput was $0.282 \mathrm{Kbit} / \mathrm{sec}$, and it was appropriate for the transmission of real-time multimedia. But AODV routing mechanism saves the minimum hop count routing only, when the connection is interrupted, AODV has to redo the routing search and that will increase the network load. Researches in recent years has improved the overall performance of AODV [13]. K. S. Kumar et al. [14] introduced the method of Bayesian classifier and fuzzy proximity relation to classifier the MANET and predict the movement of nodes, and it could get a more efficient routing mechanism. In AOZDV (Ad hoc Ondemand Zone and Distance-Vector Routing), H. Lee et al. [15] divided the network into several clusters. Each cluster adopts AODV and using a table-driven routing mechanism based on distance 
vector among cluster head nodes. This scheme decreased the routing searching time in large scale network and lower the routing loss rate. R. Bai et al. [16] combined the DSR and AODV, and adopted a lightweight network classifier model, i.e. stratify the active nodes into several layers. The method fits the transmission in large scale network. The above researches had improved the performance of AODV, but it cannot satisfy the requirement of QoS in the routing scene under the end-to-end real-time constraint in MANET network.

\section{Proposed Scheme - LL-AODV}

The conventional AODV and exiting revised AODV cannot support the end-to-end real-time communication, this paper proposed a novel revised AODV routing mechanism, named LL-AODV (Ad-hoc on-demand distance vector routing based on location information and link-stability evaluation).

LL-AODV uses the same mechanism of AODV in the routing discovery, but it changes the content of the RREP returns and the adjusts the routing selection. LL-AODV draws on the design of OSPF, which source node maintains a simplified database of link state, and introduces three metrics (i. e. the duration time of links, the residual battery and energy consumption of nodes) to calculate the weight of links. LL-AODV abstracts the QoS routing problems into the shortest path problem of an undirected weighted graph and adopts two types routing maintenance mechanism: periodical maintenance and rescue maintenance. LL-AODV modify deficiency of AODV which only selected route based on hop count. The implementation of LL-AODV ensures the quality of routing selection and reduce the possibility of communication outage. The introduction of the periodical routing maintenance allows node set the routing maintenance classes and the rescue routing maintenance keeps the network expedite when link interruption occurs, and thus guarantees the real-time feature of the transmission and the effectiveness of paths.

The work flow of LL-AODV is as follows:

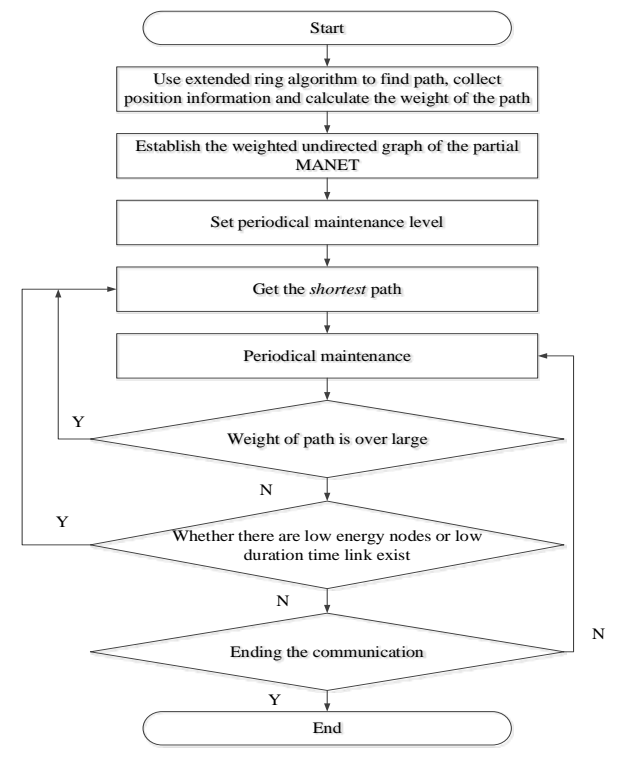

Fig. 1. The working flowchart of LL-AODV.

\section{A. Routing Establishment}

\section{1) Acquirement of the position information}

Source node broadcasts RREQ control packet to its neighbor node and the nodes will establish or update a reserve routing when a non-repetitive RREQ packet is received. The speed and the coordinates of the nodes can be obtained by utilizing Global Position System (GPS). All nodes pick up the RREQ packet and broadcast it until the destination node or the intermediate node which has a valid routing access to the destination node receives the packet. Then the destination node or the intermediate node will reply a RREP packet to source along the reverse path. Source node takes 
advantage of expanding ring search algorithm to expand the broadcasting area gradually, and it will achieve a better trade-off between routing overhead and the effectiveness of routing lookup.

\section{2) Parameters collection}

LL-AODV revises the content of the EERP packet. A revised RREP packet includes information like the coordinates and the instantaneous velocity of nodes, the energy consumption level of nodes, and the current weighted energy of nodes, here, the coordinates and the velocity of nodes can be obtained through GPS. And these data are the basis of the following route selection.

\section{3) Establishing undirected graph}

After all the RREP packets return, the source node will establish an undirected graph $\mathrm{G}$ based on the content contained in RREPs. And the contains information used by the source node including the position of the nodes and the effective range of the devices. The undirected graph $G$ is a localized network of the MANET in this conversation. And the following illustration shows the model of the undirected graph.

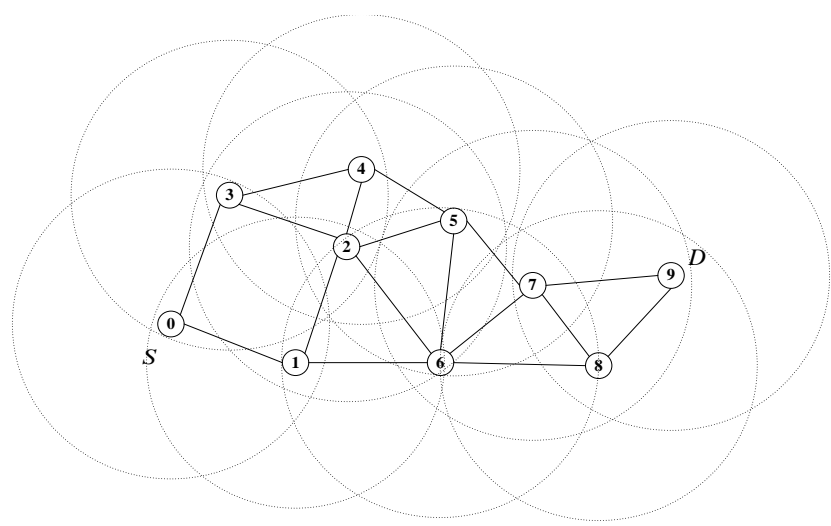

Fig. 2. The model of the undirected graph.

\section{4) Weight calculation}

\section{a)Prediction of duration time of links $t_{i j}$}

The duration time that the two mobile nodes will remain connected can be predicted by utilizing the following prediction model of the duration time of links [17]. Assume we have two nodes ${ }^{i}$ and $j$, and they have the equal transmission range $T x .\left(x_{i}, y_{i}\right)$ and ${ }^{\left(x_{j}, y_{j}\right)}$ are the coordinate of node $i$ and $j$, respectively. ${ }^{v_{i}}$ and ${ }^{v_{j}}$ is the speed of node ${ }^{i}$ and ${ }^{j}$, and let $\theta_{i}, \theta_{j}$ be the headings of node ${ }^{i}$ and ${ }^{j}$, respectively. Then ${ }^{t_{i j}}$ is the duration time that they will remain connected.

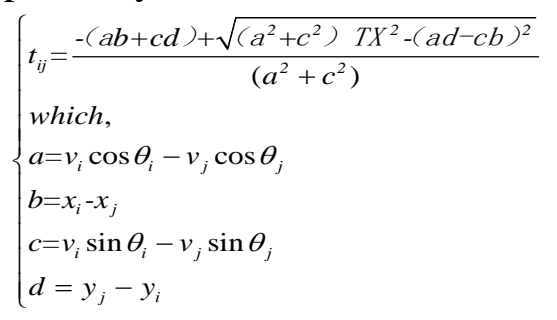

The large value of ${ }^{t_{i j}}$ means the greater connection between nodes, and the link will be stranger. When ${ }^{t_{i j}}$ diminishes, the stability of link diminishes as well. The link will go down at any time, and the maintenance mechanism need to be fully prepared.

\section{b)Weighted power of nodes $p_{i j}$}

The remaining capacity of battery has a direct impact to the working hours of nodes, and the link transmission time is determined by node with a lower energy. We introduce the weighted power of nodes here and set a threshold of the energy. And the weighted power of node ${ }^{p_{i j}}$ is given by:

$$
p_{i j}=\operatorname{Min}\left(P_{i}, P_{j}\right) / P_{o}
$$

which $P_{i}$ and $P_{j}$ are the remaining energy of node $i$ and $j$, respectively, and $P_{o}$ is the threshold. 


\section{c) Energy consumption of nodes $E_{i j}$}

The energy consumption of nodes will indirectly affect the future working time. It is assumed that the wireless channels are totally symmetrical, i.e., when the signal to noise ratio is invariable, the energy consumption of node ${ }^{i}$ sends ${ }^{k}$ bit data to node ${ }^{j}$ equals the energy consumption of node ${ }^{j}$ sends bit data to node ${ }^{i}$. In wireless network model, the total energy consumption mainly contains the circus energy consumption $E_{\text {elec }}$, transmission energy consumption ${ }_{E_{5}} d^{2}\left({ }^{\varepsilon_{m p}} d^{4}\right)$, and receiving energy consumption [18]. The value of the circuit energy consumption $E_{\text {elec }}$ are on the base of the electrocircuit hardware.

Based on the relationship between the transport distance $d$ and the distance threshold ${ }^{d_{0}}$, wireless channel models can be divided into two categories: free space channel model and the multipath fading channel model. If $d$ is larger than ${ }^{d_{0}}$, multipath fading channel model should be applied, and if $d$ is lower than ${ }^{d_{0}}$, free space channel model should be applied. $\varepsilon_{i s} d^{2}$ and $\varepsilon_{m p} d^{4}$ represent energy consumption parameters of relevant amplifiers of two models, respectively. Therefore, when a ${ }^{k}$ bit data need to be send, the transmission energy consumption $E_{T x}(k, d)$ and the receiving energy consumption $E_{R x}(k)$ are given by:

$$
\begin{aligned}
& E_{T x}(k, d)=E_{T x-\text { elec }}(k)+E_{\text {Tx-anp }}(\mathrm{k}, \mathrm{d})=\left\{\begin{array}{l}
k\left(E_{\text {elec }}+\varepsilon_{f s} d^{2}\right),\left(d<d_{0}\right) \\
k\left(E_{\text {elec }}+\varepsilon_{m p} d^{4}\right),\left(d \geq d_{0}\right)
\end{array}=\left(\beta_{1}+\beta_{2} d^{n}\right) k\right. \\
& E_{R x}(\mathrm{k})=E_{R x-\text { elec }}(\mathrm{k})=k E_{\text {elec }}=k \gamma_{1}
\end{aligned}
$$

When the circuit is fixed, $E_{\text {elec }}$ is a constant. And we can see from (3) (4) that the consumption of sending date and the consumption of receiving data are proportional to the amount of data, i.e., the larger the data to be sent, the higher the energy consumes. And the transmission energy consumption is proportional to $d^{2}$ or $d^{4}$, i.e., as distance increases, the energy consumption will increase rapidly. And when the distance achieves a high value, the transmission energy consumption will play a large part in the total energy consumption.

Thus, to get the minimum energy consumption and the longest processing time, the model is given by:

$\operatorname{Min}(E)$

$E=E_{T}+E_{R}+E_{o}$

which, $E_{T}$ is the transmission energy consumption, $E_{R}$ is the receiving energy consumption and $E_{o}=\alpha E_{\text {elec }}$ is the idle energy consumption. If the amount of the data to be sent is fixed, the receiving energy consumption and the idle energy consumption will remain unchanged, but the transmission energy consumption will vary depending on the transport distance. Therefore, the transmission energy consumption is the most critical element to the energy consumption, and the simplified model is:

$\operatorname{Min}\left(E_{T}\right)$

In formula (3), $k$ is the total bytes transferred, $E_{\text {elec }}$ is the circus energy consumption, $\varepsilon$ is the energy consumption parameter of amplifier, and they are all constant. Thus, the model can be simplified as:

$\operatorname{Min}\left(d^{n}\right)$

which the value of $n$ for 2 or 4 , and topically, set ${ }^{n=2}$. And the value of $d$ can be measured by the GPS.

\section{d)Weight calculation}

LL-AODV introduces multiple variables as the measurement criteria for path selection. The introducing of the duration time of links can avoid the effect of the unstable routing caused by the movement of the nodes. The weighted power of nodes avoids the communication termination caused 
by low power of equipment. And the energy consumption can be used to avoid the duration problems caused by excessive energy consumption of nodes. The total weight of links is given by:

$w_{i j}=w_{j i}=a_{1} / t_{i j}+a_{2} / p_{i}+a_{3} E_{i j}$

which, ${ }^{a_{1}}, a_{2}, a_{3}$ are the proportional coefficients for parameters, and $a_{1}+a_{2}+a_{3}=1$. The longer duration time of link, the higher weighted power and the lower energy consumption, and the lower the total weight.

\section{5) Path Selection}

After all the weights of links have been calculated, we could build an undirected weighted graph. The following illustration shows the model of the graph.

The mechanism uses the shortest path algorithm to calculate the shortest path between the source node and the destination node. And this path is also the fittest path which best meets the requirements of QoS.

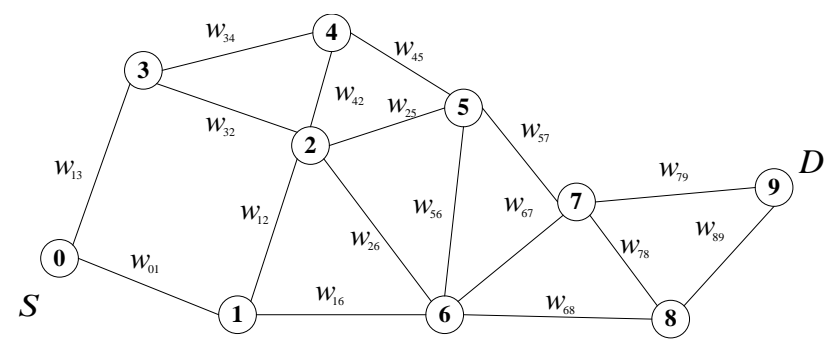

Fig. 3. The model of the undirected weighted graph.

\section{B. Route Maintenance}

Based on the using scale, there are two kinds of route maintenance [19][20] mechanisms: network maintenance and path maintenance.

The network maintenance mechanism is used to maintain the entire MANET network. In LLAODV, it is a periodical maintenance. Using the expanding ring search algorithm, it measures the state change and movement of nodes and update the information of each node in MANET periodically. Then it will update the database of link state.

For path maintenance [21][22], it divided into two parts: periodical maintenance and rescue maintenance.

- Periodical route maintenance. At the initial stage, the source node will set a periodicity level of this communication based on the requirements of QoS, and then calculates the parameters of the existing paths. When the overall weight of the main path is larger than threshold weight, the LL-AODV will be activated and reselect another better path.

- Rescue route maintenance. When the weighted power of node is lower than the given threshold or the duration time of link is lower than the routing maintenance period, the shortest path algorithm begins to reselect a good path. The using of the rescue maintenance mechanism could avoid the transmission interruption when node goes down or cannot provide the transport service due to the change of the location.

\section{Performance study with simulation}

In this section, we make a performance evaluation and comparison between the revised AODV (LL-AODV) and the conventional AODV routing protocol in the real-time multimedia data transmission. MANET is regarded as a collection of mobile nodes. Each node changes its location dynamically and the interconnections between nodes change based on a continual basis. As nodes belonging to MANET may move arbitrarily and unpredictably, the network shoud be able to react to the frequent topological changes. This paper uses Ns-2 to simulate this test environment. 


\section{A. Simulation Environment}

TABLE I. SIMULATION PARAMETERS

\begin{tabular}{|l|l|}
\hline \multicolumn{1}{|c|}{ Parameter } & \multicolumn{1}{c|}{ Table Column Head } \\
\hline Copy & LL-AODV, AODV \\
\hline Dimension & $600 \mathrm{~m} \times 600 \mathrm{~m}$ \\
\hline Number of nodes & 50 \\
\hline Max speed of nodes & $2,5,10,15$ meters/second \\
\hline Initial energy of nodes & $35,24,12$ Joules \\
\hline Threshold energy of nodes & 4 Joules \\
\hline Packet size (non-video packets) & 512 Bytes \\
\hline Channel & Wireless Channel \\
\hline Simulation duration & 100 seconds \\
\hline
\end{tabular}

In this simulation, each node changes its position based on a random model. And there are fifty nodes randomly deployed in a square area. At the beginning of the simulation, each node selects a random distance and moves to it with a constant speed. When nodes arrive to their distance, they will take a random pause time to be quiescent, and then move to another random selected distance with the same speed. And this movement will continue over the entire simulation phase. The speeds of nodes are set between 0 to $15 \mathrm{~m} / \mathrm{s}$. And the pause time of each node is set randomly between 0 to 12 seconds. Among these fifty nodes, 20 nodes have a high initial energy, 18 nodes have the medium initial energy and the rest with the low initial energy. And the simulation system sets up a threshold energy as the end tag of nodes. When the energy of node falls below the threshold, the node will stop working and become dormant. What's more, in order to enable the fairness of the comparisons between routing protocols, we used the same initial node position for each simulation. And the test media of this simulation is divided into two part: audio and video, and they are specially designed for certain metrics. Table I summarizes the simulation parameters.

\section{B. Evaluation Metrics}

The evaluation metrics are in terms of the node failure ratio, average delay and frame loss rate. And we adopt real-time audio stream as the test media for the node failure ratio and average delay, and each packet is below 512 bytes. For the frame loss rate, we use a video file which has 500 frames. It contains $18 \mathrm{I}$ frames and $482 \mathrm{P}$ frames.

The node failure ratio ${ }^{R_{N F} \%}$ refers to the ratio of energy-exhausted nodes ${ }^{N_{F}}$ to all nodes ${ }^{N_{T}}$ (the energy-exhausted nodes mean the energy of nodes are below the threshold energy), and it reflect the state of the network.

$$
R_{N F} \%=\frac{N_{F}}{N_{T}} \times 100
$$

The average delay refers to short period of time for all date packets between when an audio packet generated by source and when it replayed by the destination. In our simulation, average delay is a statistical average of twenty experimental results.

Frame loss rate describes the loss rate that will be seen by the transport protocols. For each kinds of frame, we measure the lost frames separately, and the percentage of the total frame loss can be calculated as:

$$
L_{T F} \%=\left(1-\frac{F_{R I}+F_{R P}}{F_{T}}\right) \times 100
$$

which, $L_{T F} \%$ represents the percentage of the total frame loss, $F_{R I}$ is the received number of I frame and $F_{R P}$ is the received number of $\mathrm{P}$ frame, $F_{T}$ represents the total number of the frame the source sends during the simulation phase. And the percentage of the I frame loss can be calculated as:

$$
L_{I F} \%=\left(1-\frac{F_{R I}}{F_{T I}}\right) \times 100
$$


which, $L_{I F} \%$ represents the percentage of the I frame loss and $F_{T I}$ represents the total number of the I frame the source sends during the simulation phase. The percentage of the total frame loss and the I frame loss shows the characteristic of the routing protocol both in completeness and correctness.

\section{Experimental Results}

During the simulation phase, in order to evaluate the node failure ratio, we identified the number of the shutting-down nodes every 5 seconds. Fig. 4 to 7 separately illustrate the results of node failure ratio with nodes moving in maximum $2 \mathrm{~m} / \mathrm{s}, 5 \mathrm{~m} / \mathrm{s}, 10 \mathrm{~m} / \mathrm{s}$ and $15 \mathrm{~m} / \mathrm{s}$. Fig. 8 shows the average delay in which nodes move with speed of $2 \mathrm{~m} / \mathrm{s}, 5 \mathrm{~m} / \mathrm{s}, 10 \mathrm{~m} / \mathrm{s}$ and $15 \mathrm{~m} / \mathrm{s}$, respectively. And Fig. 9 represents the Average I frames loss rate and total frames loss rate in different maximum moving speed. During the entire simulation period, all the transmissions are real-time.

The results show that, compared with the conventional AODV routing protocol, LL-AODV's node failure ratio is relatively high (see Fig. 4 to 7). That means LL-AODV possesses a higher dissipation capacity than conventional AODV. The reason is that, in real-time transmission, the conventional AODV uses the first arrived RREQ with the minimum hop count to generate the transport route, however, the LL-AODV needs to measure and collect the parameters of nodes and links, and thus will consume part of the nodes energy. And the LL-AODV adopts periodicity maintenance and rescue maintenance to perform the routing maintenance, which will bring additional energy consumption. In LL-AODV, lower nodes energy means a higher weight, and the selected probability of the nodes will be lower. And on the other hand, the load balance will be achieved when the node with a good performance will be chosen as the primary choice.

In Fig. 8, LL-AODV reduces the average delay during the simulation phase. Because in AODV, the transport path which was built by the first arrival RREQ may be not stable and will go down at any time, lacking routing maintenance mechanism, the path is not suit for real-time media transport. Paths generated by the LL-AODV are more stable and the routing maintenance mechanism will enhance the effectiveness of the transport paths.

Video date transport means a heavy traffic and a high load of network. In Fig. 9, with the introduction of heavy traffic in simulation, LL-AODV protocol gives the best performance while conventional AODV gives the worst performance. The main reason is that the path built by the conventional AODV is not the optimal, and the path quality cannot be guaranteed. With the network load increasing, the potential of path meltdown will also increase. However, LL-AODV adopts the best path to transmit data, when the load of network is increased, the weight of the relevant path will increase, and LL-AODV will reselect a new path with the minimum weight based on the local network information. Therefore, the data will be transmitted over a reliable path. 


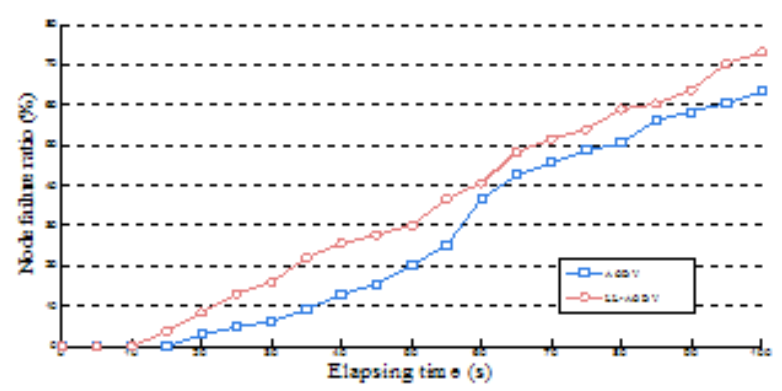

Fig. 4. Node failure ratio comparison for maximum moving speed $2 \mathrm{~m} / \mathrm{s}$.

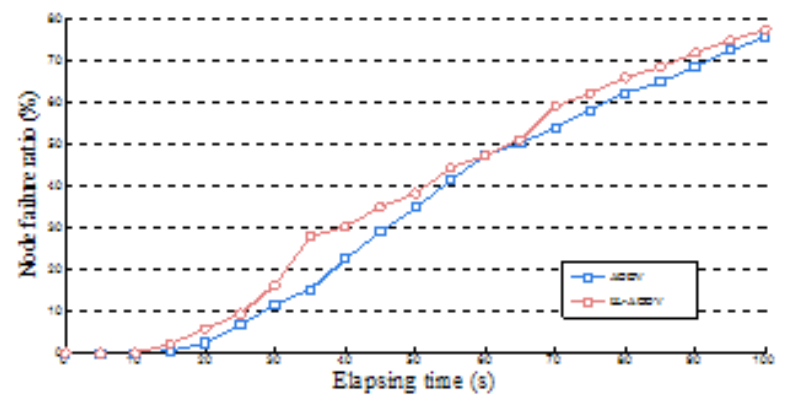

Fig. 6. Node failure ratio comparison for maximum moving speed $10 \mathrm{~m} / \mathrm{s}$.

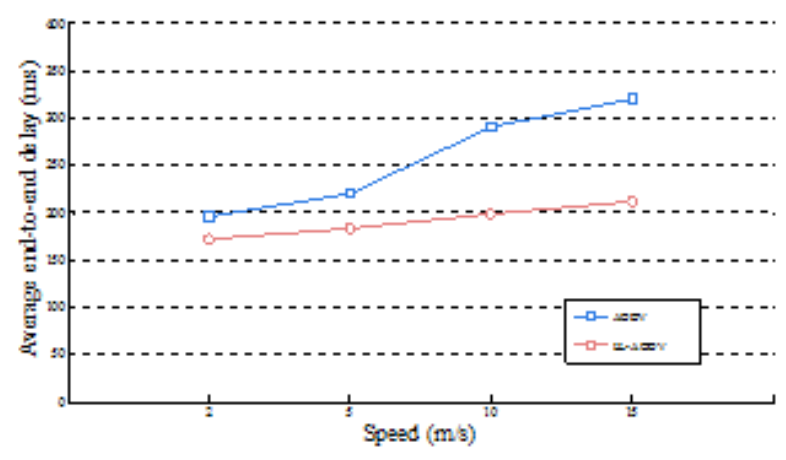

Fig. 8. Average end-to-end delay in different maximum moving speed.

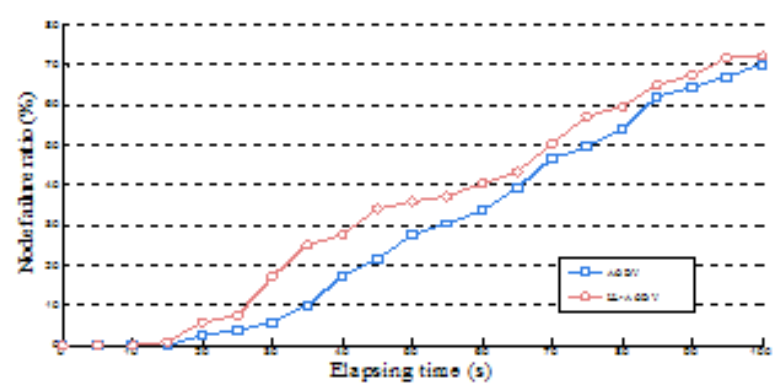

Fig. 5. Node failure ratio comparison for maximum moving speed $5 \mathrm{~m} / \mathrm{s}$.

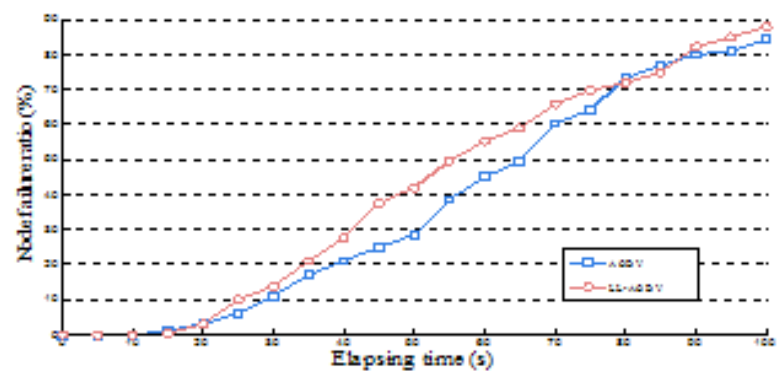

Fig. 7. Node failure ratio comparison for maximum moving speed $15 \mathrm{~m} / \mathrm{s}$.

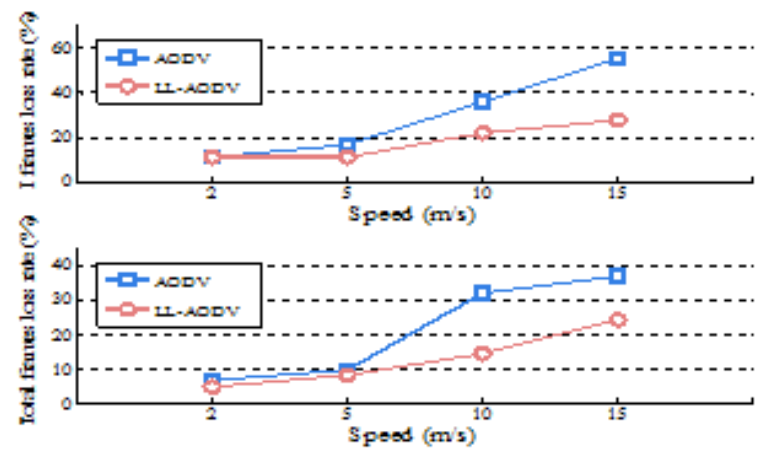

Fig. 9. Average I frames loss rate and total frames loss rate in different maximum moving speed.

\section{Conclusion}

In this paper, we proposed a novel AODV routing mechanism based on the location information and link-stability evaluation. This mechanism abstracts the routing problems into the shortest path problem of an undirected weighted graph and adopts the shortest path algorithm to select the best route. The mechanism uses the duration time of links, the residual battery and energy consumption of nodes as the evaluation indexes and could provide a better QoS routing for end-to-end real-time communication in MANET. The mechanism improves the conventional routing mechanism in some extent, such as the reliability of paths, network load balance, etc. Despite the increased overhead, the implementation of this mechanism has reduced the packet loss rate and enhanced the robustness of paths, and in the aspect of end-to-end delay, the mechanism can provide a good performance. Future work will focused on the energy reduction of nodes and the assignment of the weights of the metrics to guarantee the scientific nature of route selection.

\section{Acknowledgment}

This work was supported by the National Training Program of Innovation and Entrepreneurship for Undergraduates (No.201510145062) ,National Natural Science Foundation of China (No.61401081) and Fundamental Research Funds for the Central Universities (No. N150404005). 


\section{References}

[1] S. Corson and J. Macker, "Mobile Ad hoc Networking (MANET): Routing Protocol Performance Issues and Evaluation Considerations,” RFC 2501, Jan. 1999.

[2] I. Chalmtac, M. Conti, J. Liu, "Mobile Ad hoc Networking: Imperatives and Challenges”, Elsevier Journal on Ad hoc Networks, vol. 1, no. 1, 2003, pp. 13-64.

[3] A. Moussaoui, A. Boukeream, "A survey of routing protocols based on link-stability in mobile ad hoc networks”, Journal of Network and Computer Applications, vol. 47, 2015, pp. 1-10.

[4] J. Hoebeke, I. Moerman, B. Dhoedt, and P. Demeester, "An overview of mobile ad hoc networks: Applications and challenges”, Journal of the Communication Networks, vol. 3, no. 1, 2004, pp. 60-66.

[5] X. Hong, K. Xu, M. Gerla, “Scalable routing protocols for mobile ad hoc networks”, IEEE Network. Vol. 16, no. 4, 2002, pp. 11-21.

[6] A. Boukerche, B. Turgut, N. Aydin, M. Z. Ahmad, L. Bölöni, D. Turgut, "Routing protocols in ad hoc networks: A survey”, Comput. Netw. vol. 55, no. 13, 2011, pp. 3032-3080.

[7] E. Alotaibi, B. Mukherjee, "A survey on routing algorithms for wireless ad-hoc and mesh networks”, Comput. Netw. vol. 56, no. 2, 2012, pp. 940-965.

[8] M. A. Mojamed, M. Kolberg, "Structured Peer-to-Peer overlay deployment on MANET: A survey”, Comput. Netw. vol. 96, 2016, pp.29-47.

[9] M. Abdel-Hady, R. Ward, “A Framework for Evaluating Video Transmission over Wireless Ad Hoc Networks”, Proc. 2007 IEEE Pacific Rim Conf. Communications, Computers and Signal Processing (PacRim 2007), 2007, pp. 78-81.

[10] T. Huang, W. Chung, and C. Huang, “A Revised AODV Protocol with Energy Management for Real-Time/Non-Real-Time Services in Mobile Ad Hoc Network”, Proc. The 10th IEEE International Conf. High Performance Computing and Communications (HPCC '08), 2008, pp. 440446.

[11] C. E. Perkins, E. M. Royer, "Ad-hoc on-demand distance vector routing”, Proc. IEEE Workshop on Mobile Computing Systems and Applications (WMCSA '99), 1999, pp. 90-100.

[12] T. Setiadi, Y. Bandung, "Performance evaluation fo real-time multimedia package in multihop wireless network with AODV protocol", Proc. 2012 7th International Conf. Telecommunication Systems, Services, and Applications (TSSA), 2012, pp. 24-27.

[13] Z. Mou, X. Meng, “A modified AODV routing protocol based on route stability in MANET”, Proc. 4th IET International Conf. Wireless, Mobile \& Multimedia Networks (ICWMMN 2011), 2011, pp.63-67.

[14] K. S. Kumar, T. Arunkumar, "Classification and prediction of routing nodes behavior in MANET using Fuzzy proximity relation and ordering with Bayesian classifier”, Proc. 2013 International Conf. Pattern Recognition, Informatics and Mobile Engineering (PRIME), 2013, pp. 454-460.

[15] H. K. Lee, Y. W. Kim and J. S. Song, “AOZDV: An enhanced AODV protocol based on zone routing in MANET”, Proc. 2007 International Conf. Wireless Communications, Networking and Mobile Computing (WiCom 2007), 2007, pp. 1685-1688.

[16] R. Bai, M. Singhal, "DOA: DSR over AODV Routing for Mobile Ad Hoc Networks", IEEE Transactions on Mobile Computing, vol. 5, no. 10, 2006, pp. 1403-1416. 
[17] W. Su, M. Gerla, "IPv6 flow handoff in ad hoc wireless networks using mobility prediction", Proc. IEEE GLOBECOM'99, 1999, pp. 271-275.

[18] H. B. Wendi et al., "An Application-Specific Protocol Architecture for Wireless Microsensor Networks", IEEE Transactions on Wireless Communications, vol. 1, no. 4, 2002, pp. 660-670.

[19] S. Park et al., ARM: Anticipated Route Maintenance Scheme in Location-Aided Mobile Ad Hoc Networks, Journal of Communications and Networks, vol. 7, no. 3, 2005, pp. 325-336.

[20] G. Jisha and P. Samuel, Coverage Based Route Maintenance in MANET Routing Protocol, Proc. 2012 International Conf. Intelligent Systems Design and Applications (ISDA), 2012, pp. 398 403.

[21] L. Tang and H. Wang, Performance Analysis of Route Maintenance of MANET, Proc. IET Conf. Wireless, Mobile and Sensor Networks (CCWMSN 07), 2007, pp. 133-137.

[22] P. Srinivasan and P. Kamalakkannan, "Enhancing Route Maintenance in RSEA-AODV for Mobile Ad Hoc Networks", Proc. 2013 7th International Conf. Intelligent Systems and Control (ISCO), 2013, pp. 464-469. 